\title{
A Modified Leach Routing Algorithm for Increasing Lifetime of the Wireless Sensor Network
}

\author{
Amer Abu Salem \\ Zarqa University \\ Jordan \\ abusalem@zu.edu.jo
}

ABSTRACT: Wireless sensor networks (WSNs) are composed of many low cost, low power devices with sensing, local processing and wireless communication capabilities. The major limitation of wireless sensor network is energy and network lifetime. In this paper, the cluster routing protocol LEACH (Low-Energy Adaptive Clustering Hierarchy) is considered and enhanced.

This paper proposes a clustering routing protocol named Enhanced LEACH, which extends LEACH protocol by selecting a cluster head according to the minimum distance to the base station, which plays an important role in reducing power consumption in cluster head nodes and in the overall network.

The simulation results show that Enhanced LEACH outperforms LEACH in terms of network lifetime and power consumption minimization.

Keywords: Wireless sensor networks, LEACH, Enhanced LEACH

Received: 18 April 2018, Revised 28 May 2018, Accepted 4 June 2018

DOI: $10.6025 / \mathrm{jio} / 2018 / 8 / 3 / 114-123$

(C) 2018 DLINE. All Rights Reserved

\section{Introduction}

Wireless sensor networks participate an important role in the wireless communication. Wireless sensor networks are benefits in various applications such as civil, military field, and health care applications. This network consists of sensor nodes. That is capable of sensing the temperature level, pressure condition, humidity etc. This network is used to collect data from the physical environment and process the data and transmits to the base station [1].

The demands of these networks are ease of deployment, long-lifetime system, and low-latency data transfers. The main task of a sensor node in a sensor field is to detect events, perform quick local data processing, and then to transmit the data [2].

The main components of WSN: sensing unit, Communication unit and processing unit [3]. Sensing unit; which consists of

$114 \quad$ Journal of Information Organization Volume 8 Number 3 September 2018


sensors and analog digital converter (ADC), Sensors equipped with a battery, and this battery will expire after a time and it is hard to replace it in some application that is used for such as: in homeland defense, military surveillance, and environmental sensing, and thus a WSN has a limitation of energy ,such the consumption of energy in a WSN fall in sensing, computing, and communicating [4].the ADC in sensing unit is used for convert a signal from analog to digital by ADC. Sensors sense physical or environmental phenomena and generate an analog signal then ADC is used to convert this signal to digital signal. The second main component is the communication unit; it is responsible for data transmission and reception over a channel. In addition, the third component is the processing Unit: Processing Unit consists of microcontroller and microprocessor, which it provides a control to the sensor node.

In direct communication WSN, the sensor nodes directly transmit their sensing data to the Base Station (BS) without any coordination between the two. However, in Cluster-based WSNs, the network is divided into clusters, and this technique is called clustering. Clustering means the division of data into groups of similar object. In a WSN with a large amount of energy constrained sensor it is important to organize sensors into clusters to reduce the energy consumption, scalability, prolong the network lifetime, reduce the delay, and handle the heterogeneity of network.

Each sensor node exchanges its information only with its cluster head $(\mathrm{CH})$, which transmits the aggregated information to the BS. Aggregation and fusion of sensor node data at the CHs because a significant reduction in the amount of data sent to the BS and so results in saving both energy and bandwidth resources. On the other hand, clustering is particularly crucial for scaling the network to hundreds or thousands of nodes [5]. In many applications, cluster organization is a natural way to group spatially close sensor nodes in order to exploit the correlation and eliminate the redundancy that often shows up in the sensor readings [6]. However, these benefits, compared to those of the direct communication WSN, result in extra overhead due to the cluster formation's message exchanges.

There are some challenges while transmitting the sensed information in the networks or outside the network when using a cluster based routing protocol; such as: some cluster based routing protocol are suitable for small regions or small number of nodes only, some of cluster based routing protocol are suitable to deploy a node in static manner thus it is not suitable for mobile nodes, in some cluster based routing protocols the distribution of cluster head is concentrated to one area only, some cluster based routing protocol is not suitable for time critical application, some cluster based routing protocol allows all cluster heads to send data aggregated to base station which reduces the energy consumption [7].

\section{Leach - Low Energy Adaptive Clustering Hierarchy Protocol}

LEACH is a hierarchy routing protocol used in WSN to increase the lifetime of a network. In LEACH protocol a sensors organize themselves in a cluster and one of these nodes acts as a cluster head. Only a cluster head is allowed for send the data to the base station, cluster head collects a data from all nodes then aggregates and compress then send it to the base station. LEACH is an adaptive, self-organizing and clustering protocol. LEACH has the assumption according to sensor characteristics and base station [8].

In LEACH protocol a cluster head is not fixed, LEACH used a concept of round and each round consists of two phases: a set-up phase and steady state phase. The set-up phase divided into Advertisement Phase and Cluster Set-up Phase. While steady phase divided into Schedule Creation and Data Transmission [9].

LEACH protocol is suitable for the WSNs under the following assumptions:

o All senor nodes are static, identical and charged with the same amount of initial energy.

o All nodes consume energy at the same rate and are able to know their residual energy and control transmission power and distance.

o Every node can directly communicate with every other node, including the sink node.

o The Sink node is fixed and far away from the wireless network. Thus, we can ignore the energy consumed by the sink node.

o Every node has data to transfer in every period. The data transferred by sobering nodes are related and can be fused [10].

However, LEACH has some disadvantages such as:

Journal of Information Organization Volume 8 Number 3 September 2018 
o LEACH does not provide clarity about the position of sensor nodes and the number of cluster heads in the network.

o Each Cluster-Head directly communicates with BS no matter the distance between $\mathrm{CH}$ and BS. It will consume a lot of its energy if the distance is far.

o The $\mathrm{CH}$ uses most of its energy for transmitting and gathering data, because, it will die faster than other nodes.

o The $\mathrm{CH}$ is always on and when the $\mathrm{CH}$ die, the cluster will become useless because the data collected by cluster nodes will never reach the base station [11].

\section{Related Work}

Power consumption is the main concern in developing wireless sensor networks applications. Consequently, several strategies have been introduced for estimating the power dissipation of this kind of application. These strategies can help to predict the WSN lifetime, give recommendations to application developers and may optimize the energy dissipated by the WSN applications.

Heinzelman, et al. (2000) proposed a clustering algorithm for sensor networks, called Low Energy Adaptive Clustering Hierarchy $(\mathrm{LEACH})$. LEACH forms clusters by employing a distributed algorithm, where nodes produce independent decisions without any centralized control. LEACH organizes the nodes in the network into clusters and selects one of them as $\mathrm{CH}$. The operation procedure of LEACH divided into rounds. Each round starts with a setup phase when the clusters are organized, the second is a steady-state phase when data is transmitted from nodes to the $\mathrm{CH}$ and then to the BS [12].

LEACH-E (Energy Low Energy Adaptive Clustering Hierarchy) presents a new approach to defining lifetime of sensor in that all a node have the same energy and same probability to become a cluster head then, after the first round the residual energy will be change and the cluster head will the nodes with high residual energy thus the nodes themselves determine whether they become cluster-heads. Communication with the base station is not necessary [13].

Kumar et al. (2011) Discussed an LEACH-F (Fixed number of cluster Low Energy Adaptive Clustering Hierarchy) this protocol uses centralized approach for cluster formation. Once the cluster formation process created, then there is no need to reclustering phase in next round. The clusters topology is fixed, and only rotate cluster head nodes within its clusters; the steady phase is the same as classical LEACH. The advantages of this protocol are that re-clustering not needed once the fixed number of clusters formed; they are maintained throughout the network. The disadvantages of this protocol provide no flexibility of adding or removing the nodes once clusters formed and nodes cannot adjust their behavior on node dying [14].

Sindhwani, and Vaid, (2013) introduces Vice Cluster Head Low Energy Adaptive Clustering Hierarchy which improves the drawback in LEACH protocol; by having vice-cluster head in each cluster that takes the role of cluster head when cluster head dies, this reduces overhead of selecting new cluster head each time when a cluster head dies and the data will always reach to the base station and causes increasing a life time of a network [15].

Abdellah, and Hssane (2010) Proposed Advanced Low Energy Adaptive Clustering Hierarchy A-LEACH is an extension of the LEACH, which improves the stable area of the clustering hierarchy and reduces the probability of failure nodes employing the particular parameters of heterogeneity in networks. In these networks some high energy nodes called CAG nodes become cluster head to collect the data of their cluster members and transfer it to the sink or Gateways to minimize the energy consumption of cluster head because it is employed to route information from cluster head to the sink, which permits to minimize the failure probability of clusters head and this increase the lifetime of the network [16].

Farooq et al. (2010) Multi-hop Routing with Low Energy Adaptive Clustering Hierarchy partitions the network into different layers of clusters and in each layer there is a cluster head and then cluster head collaborates with adjacent layers to transmit sensor's data to the base station. MH-LEACH adopts an optimal path between cluster head and base station [17].

Liao, and Zhu (2013) shows that An Energy Balanced Clustering Algorithm Based on LEACH Protocol depend on the residual energy and distance factors, and these improve cluster-head election and the strategy of non-cluster head node Selecting the optimal cluster-head [18]. 
Bakaraniya and Mehta (2013) K-LEACH, to prolong the lifetime of a sensor network by regular clustering through a k-medoids algorithm and balancing the capacity of the entire network among all active nodes. It guarantees regular clustering of nodes and gives the suitable location of $\mathrm{CH}$. It uses the combination of clustering, maximum residual energy criterion and a random chosen of CHs only after almost $50 \%$ of rounds of operations of the network gets over, whereas the LEACH protocol makes totally random selection of $\mathrm{CHs}$, which leads to very poor chosen of $\mathrm{CHs}$ and consequently leads to highly inefficient lifetime and energy retention by the network [19].

Kole et al. (2014) Distance Based Cluster Formation improve LEACH protocol to enhance network lifetime. The distance of the node from the base station is significant in cluster formation that will minimize some extra transmissions in existing LEACH protocol [20].

\section{Proposed Improvement of Leach}

The proposed approach is same as the LEACH; have two phases: setup and steady phase; but there is a difference in setup phase in such that in a setup phase all the normal nodes select a random number between zero and one, then if that number is less than or equal than threshold $(\mathrm{T}(\mathrm{n})$ ) as calculated according to next equation, the node becomes a cluster head $(\mathrm{CH})$ else a node is still ordinary node.

$$
T(n)=\left\{\begin{array}{cc}
\frac{p}{1-p[r * \bmod (1 / p)]} & n € G \\
0 & \text { Otherwise }
\end{array}\right.
$$

$P$ : Desired Percentage to become a Cluster head.

$r$ : Current Round.

$G$ : Set of nodes that have not been selected as Cluster head in last 1/P rounds.

That process are repeated until all cluster heads are chosen, after a cluster heads are chosen they broadcast advertisement messages overall network informing that it became a $\mathrm{CH}$. Here is a difference in LEACH in such a node must select a $\mathrm{CH}$ according to distances to reach the base station not a $\mathrm{CH}$ that is closest to it. Distances in a proposed approach calculated according to next equation; the distances calculated from a node to every $\mathrm{CHs}$ and from $\mathrm{CH}$ to base station and the minimum distances chosen.

$$
D(x, y)=\sqrt{(x 1-x 2)^{2}+(y 1-y 2)^{2}}
$$

After the ordinary node selects a cluster head that has the minimum distance, it sends a message to inform a cluster head that through it the distance is minimum, and that it will be a member of its cluster, this process is repeated to all nodes until all select appropriate cluster head for it and according to number of rounds that is required.

After receiving a request message from ordinary nodes, cluster heads create a Time-division multiple access (TDMA) schedule for each member in it. TDMA schedule assigns a time slot for every ordinary member node in it, it means that every ordinary member node is only allowed to send in its allowed time slot for it else it waits to allow time and go in sleep mode then a setup phase is completed.

Following is the algorithm for the proposed approach where the improvement shows in bold line.

After a setup phase is completed a steady phase begins, a steady phase is the same as in LEACH protocol, such that an ordinary node collects a data from surrounding environment and then sends this data (in its allowed time slot for it) to cluster heads that chosed by in the setup phase, energy consumed to transmit data through cluster head is calculated as below in Formula [21].

$$
E_{T x}(l, d)=E_{\text {elec }} * l+\epsilon_{f s} * l^{*} d^{2}
$$

Where:

$E_{T x}(l, d):$ is the energy consumed to send $l$ bit data. 
Notation:

$\mathrm{N}$ : number of nodes

$\mathrm{CH}$ : cluster head

BS: base station

\section{Setup phase:}

In this phase clusters are created ...Cluster heads are chosen For_Each $(\mathrm{N})$

$\mathrm{N}$ selects a random number $\mathrm{T}(\mathrm{n})$ between 0 and 1

If $(\mathrm{T}(\mathrm{n})<$ threshold value)

$\mathrm{N}$ become a $\mathrm{CH}$

$\mathrm{N}$ broadcasts a message advertising its $\mathrm{CH}$ status

Else

$\mathrm{N}$ become a regular node

$\mathrm{N}$ listen to advertising message of $\mathrm{CHs}$

End_If

End_for

For_Each $(\mathrm{CH})$

Calculate a distance from $\mathrm{N}$ to $\mathrm{CH}$ and from $\mathrm{CH}$ to $\mathrm{BS}$

$\mathrm{N}$ chooses the $\mathrm{CH}$ with minimum distance from it to $\mathrm{CH}$ to $\mathrm{BS}$

$\mathrm{N}$ informs the selected $\mathrm{CH}$ and becomes a member of its cluster

End_for

For_Each $(\mathrm{CH})$

$\mathrm{CH}$ creates a TDMA schedule for each node to transmit data

$\mathrm{CH}$ communicates the TDMA schedule to each node in the cluster

End_for

Algorithm 1. Setup phase algorithm

$E_{\text {elec }}:$ Energy consumed by the transmitter and the receiver circuit.

$\epsilon_{f s}:$ Amplifier parameters of transformation corresponding to the free-space technique.

$d$ : Euclidean distance between an ordinary node and cluster head as shown above.

$l$ : Packet data.

Cluster heads receive collected data from its members and runs the data compression algorithm to aggregate the collected data. Cluster heads send a compressed data to base station. The amount of energy consumed calculated by next equation [21]:

$$
E_{T x}(l, d)=E_{\text {elec }} * l+\epsilon_{m p} * l * d^{4}
$$

Where: $\epsilon_{m p}$ is the amplifier parameters of transformation correspondent to the multi-path fading model.

Pseudo code for describing the operation of the proposed steady state phase approach is below:

118 Journal of Information Organization Volume 8 Number 3 September 2018




\section{Notation :}

$\mathrm{N}$ : number of nodes.

$\mathrm{CH}$ : cluster head

\section{Steady State phase:}

For_Each (N)

$\mathrm{N}$ collects sensed data

$\mathrm{N}$ transmits the sensed data to the $\mathrm{CH}$ in the corresponding

TDMA time slot

End_for

For_Each $(\mathrm{CH})$

$\mathrm{CH}$ receives data from the nodes of the cluster

$\mathrm{CH}$ aggregates the data

$\mathrm{CH}$ transmits the data to base station

End_for

\section{Simulation Results and Analysis}

Algorithm 2. Steady state phase algorithm

There have been many protocols proposed for wireless sensor networks. In our research, we examine two such protocols LEACH protocol and the proposed approach.

Using LEACH protocol a node selects a $\mathrm{CH}$ that is closest to it regardless to distance of $\mathrm{CH}$ to base station. Using the proposed approach a node selects a minimum distance that calculated from a node to every $\mathrm{CH}$ to base station.

The following table summarizes the assumptions we have made in evaluating LEACH and the proposed approach.

\begin{tabular}{|l|l|}
\hline Parameter & Default value \\
\hline Simulation area & $100 \mathrm{~m} \times 100 \mathrm{~m}$ \\
\hline Number of nodes & $45-85$ \\
\hline $\begin{array}{l}\text { Packet Length (default packet } \\
\text { length from cluster head to } \\
\text { base station ) }\end{array}$ & 6400 \\
\hline $\begin{array}{l}\text { ctrPacket Length (default packet length } \\
\text { from normal node to cluster head) }\end{array}$ & 200 \\
\hline Initial energy & 0.5 \\
\hline Base station coordinates & $(50,50)$ \\
\hline Probability to node to become a CH & 0.1 \\
\hline Energy for transferring of each bit & $50^{*} 0.000000001$ \\
\hline Energy for receiving & $50^{*} 0.000000001$ \\
\hline Energy for free space model & $10^{*} 0.000000000001$ \\
\hline Energy for multipath model & $10^{*} 0.000000000001$ \\
\hline Energy for data aggregation & $5^{*} 0.000000001$ \\
\hline
\end{tabular}

Table 1. Simulation Parameters 
The performance of the proposed approach is compared with basic LEACH protocol in terms of average power consumption.

The following table shows a result of power consumption in cluster head nodes in LEACH and power consumption in the proposed method. In such when a number of nodes are increased power consumption in proposed approach is less because a a normal node must selects a cluster head that through it a distance is minimum so extra transmission will not occur and a cluster head locates between a normal node and a base station so a power consumption for cluster heads will reduce.

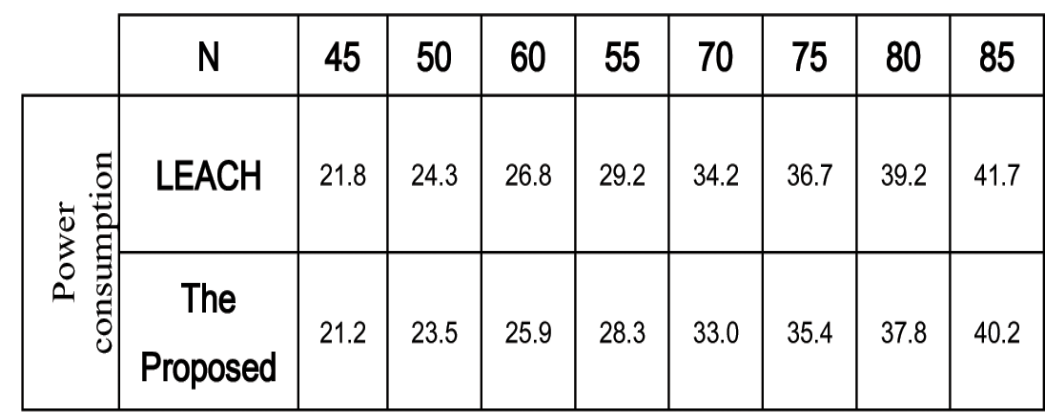

Table 3. Simulation result

As simulation started with the assumptions in Table 2, Figure 2 shows the comparison of average energy consumption at various times between LEACH and the proposed approach for different number of nodes and nine rounds.

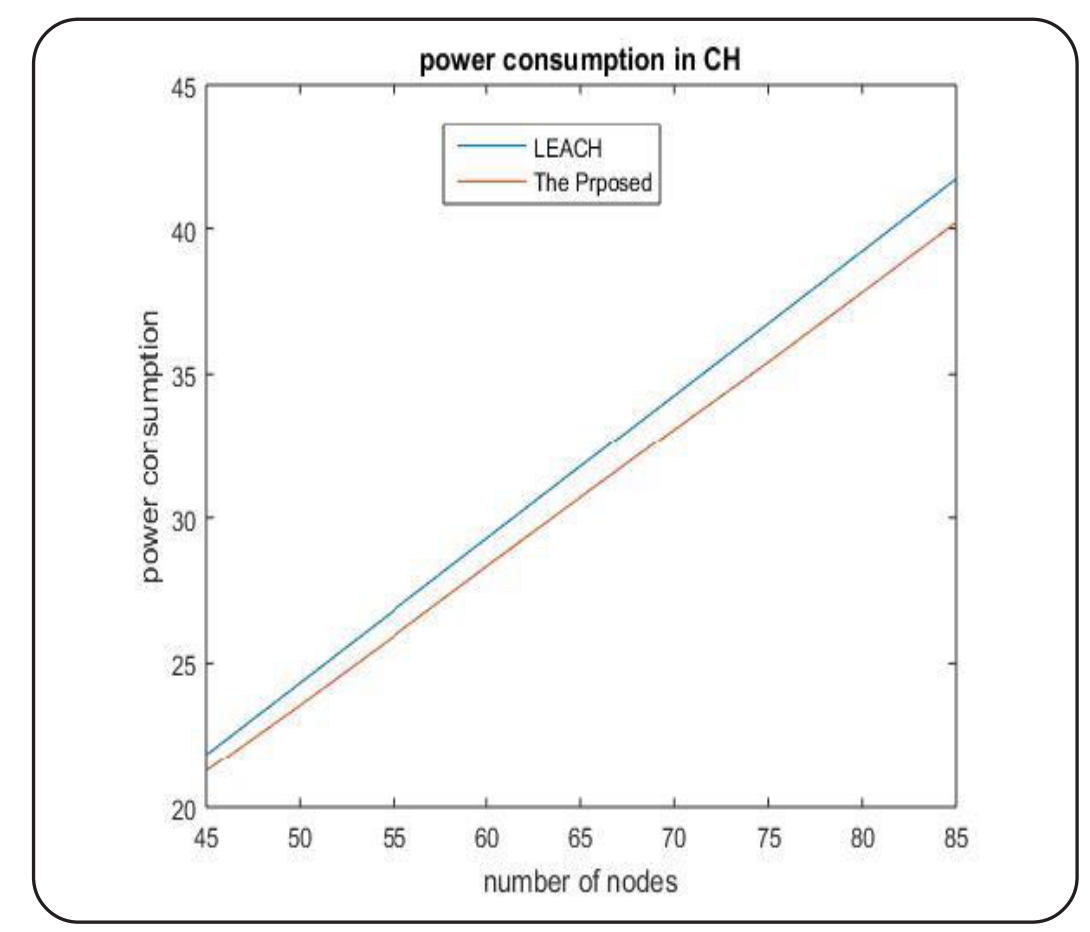

Figure 2 The comparison of average energy consumption at various times between LEACH and the proposed approach

The final extracted result plot for cluster heads shows Improvement over a LEACH protocol in such a LEACH line above the proposed line it means proposed approach consumes power less than LEACH protocol.

The below table and figure show the impact of Power consumption in overall network nodes when using LEACH Protocol with different number of nodes and nine rounds for each of them.

\begin{tabular}{lllll}
\hline 120 & Journal of Information Organization Volume 8 & Number 3 & September 2018 \\
\hline
\end{tabular}




\begin{tabular}{|c|c|c|c|c|c|c|c|c|c|}
\hline & $\mathbf{N}$ & 45 & 50 & 60 & 55 & 70 & 75 & 80 & 85 \\
\hline \multirow{2}{*}{$\begin{array}{l} \\
\dot{0} \\
0 \\
0 \\
0 \\
0 \\
0 \\
0 \\
0 \\
0 \\
0 \\
0\end{array}$} & LEACH & 22.6 & 25.1 & 30.2 & 27.6 & 35.3 & 37.8 & 40.7 & 43.2 \\
\hline & $\begin{array}{c}\text { The } \\
\text { Proposed }\end{array}$ & 22.1 & 24.6 & 29.3 & 26.9 & 34.0 & 36.4 & 39.1 & 41.5 \\
\hline
\end{tabular}

Table 3. Simulation result

As can be seen, the average energy consumption in LEACH is more than in the proposed approach with different numbers of nodes.

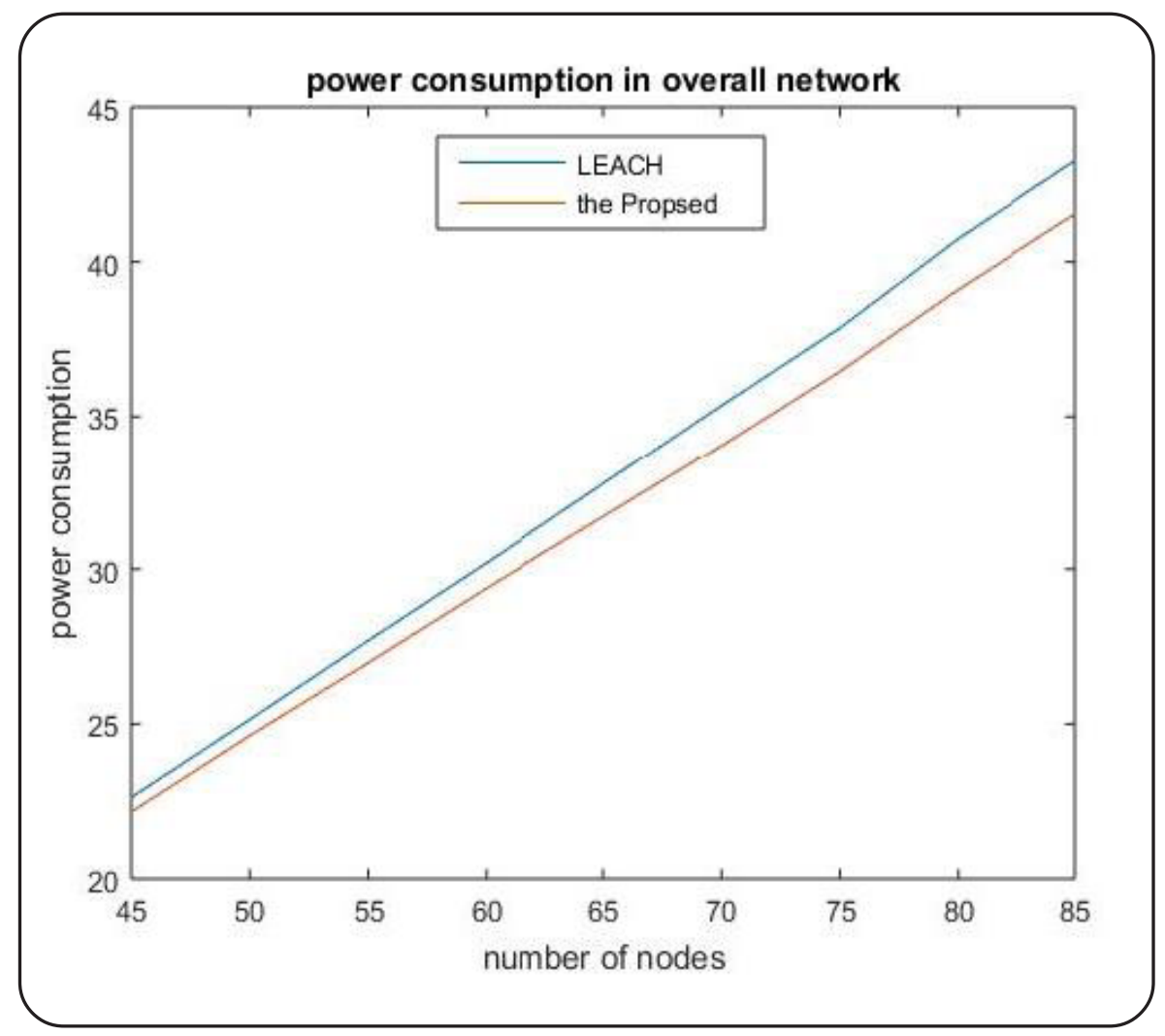

Figure 3. Power consumption in LEACH and the proposed approach

That enhancement because the improved algorithm selects cluster-head based on distances and node selects a cluster head with the distance that through it to the base station is minimum, the network energy dissipation is less thus a network lifetime will be increased.

\section{Conclusions}

A simulation based performance study was conducted to examine the power consumption of the proposed strategy from compared LEACH, and the results show that, the power consumption is reduced, and hence the lifetime of a network will be increased. As the number of rounds is increased, the reduced power consumption will be more reduced; finally, the proposed approach performs better in comparison with LEACH. 


\section{Acknowledgment}

The Deanship of Research and Graduate Studies in Zarqa University /Jordan fund this research.

\section{References}

[1] Akyildiz, I. F., Melodia, T., Chowdhury, K. R. (2007). A Survey on wireless multimedia sensor networks, Computer Networks (Elsevier) J., 51.921-960.

[2] Akiyldiz, I., Su, W., Sankarasubramaniam, Y., Cayirci, E. (2002). A survey on sensor networks, IEEE Commun. Mag., 40 (8) 102114, (August).

[3] Shahriar Mohammadi., Reza Ebrahimi Atani., Hossein Jadidoleslamy. (2011). A Comparison of Link Layer Attacks onWireless Sensor Networks, Journal of Information Security, 2. 69-84.

[4] Guobao Xu., Weiming Shen., Xianbin Wang. (2014). Applications of Wireless Sensor Networks in Marine Environment Monitoring: A Survey, Sensors 14, 16932-16954

[5] Zhu, X., Shen, L., Yum, T. P. (2009). Hausdorff Clustering and Minimum Energy Routing for Wireless Sensor Networks, IEEE Trans. Vehicular Technology, 58 (2).

[6] Soro, S., Heinzelman, W. (2009). Cluster head election techniques for coverage preservation in wireless sensor networks, $A d$ Hoc Networks, 7 (5) 955-972, (July).

[7] Yick, J., Mukherjee, B., and Ghosa, D., (2008). Wireless sensor network survey, Computer Networks 52 2292-2330.

[8] Heena Dhawan., Sandeep Waraich. (2014). A Comparative Study on LEACH Routing Protocol and its Variants in Wireless Sensor Networks: A Survey, International Journal of Computer Applications (0975 - 8887) 95 (8) (June).

[9] Leena Y. Barai., Mahendra A. Gaikwad. (2014). Performance Evaluation of LEACH Protocol for Wireless Sensor Network, International Journal of Innovative Research in Advanced Engineering (IJIRAE), 1 (6) (July).

[10] Neha Mehndiratta., Manju., Harish Bedi. (2013). Wireless Sensor Network LEACH Protocol: A Survey, International Journal of Emerging Research in Management \&Technology, 2 (3) March 2013.

[11] Meena Malik., Dr. Yudhvir Singh., Anshu Arora. (2013). Analysis of LEACH Protocol in wireless Sensor Network, 3 (2) (February).

[12] Wendi Rabiner Heinzelman, Anantha Chandrakasan., Hari Balakrishnan. (2000). Energy-Efficient Communication Protocol for Wireless Microsensor Networks, In: Proceedings of the $33^{\text {rd }}$ Hawaii International Conference on System Sciences.

[13] Handy, M., Haase, and Timmermann, D., (2000). Low Energy Adaptive Clustering Hierarchy with Deterministic Cluster-Head Selection. Fourth IEEE Conference on Mobile and Wireless Communications Networks, Stockholm, erschienen. In: Proceedings, S. 368-372.

[14] Kumar, V., Jain, S., Tiwari, S., (2011). Energy Efficient Clustering Algorithms in Wireless Sensor Networks: A Survey, International Journal of Computer Science Issues (IJCSI), 8, 4 (2) 1694-0814.

[15] Sindhwani, N., Vaid R., (2013). V Leach: An Energy Efficient Communication Protocol for WSN. Mechanica Confab 2.2, 2 (2).

[16] Abdellah, E., hssane, A., (2010). Advanced Low Energy Adaptive Clustering Hierarchy. (IJCSE) International Journal of Computer Science and Engineering 2.07, 02 (07) 2491-2497.

[17] Farooq, M., Dogar, A., Shah, G., (2010). MR-LEACH: Multi-hop Routing with Low Energy Adaptive Clustering Hierarchy. Fourth International Conference on Sensor Technologies and Applications, 262-268.

[18] Liao, Q., Zhu, H., (2013). An Energy Balanced Clustering Algorithm Based on LEACH Protocol. In: Proceedings of the $2^{\text {nd }}$ International Conference on Systems Engineering and Modeling (ICSEM-13), 5(3) 90-95, 2.

[19] Bakaraniya, P., and Mehta, S., (2013). K-LEACH: An improved LEACH Protocol for Lifetime Improvement in WSN. International Journal of Engineering Trends and Technology (IJETT) 4 (5).

[20] Kole, S., Vhatkar, K., Bag, V. (2014). Distance Based Cluster Formation Technique for LEACH Protocol in Wireless Sensor 
Network. International Journal of Application or Innovation in Engineering \& Management (IJAIEM), Volume 3, Issue 3 ISSN $2319-4847$.

[21] Ramesh, K., Somasundaram K. (2011). A Comparative Study of Cluster Head Selection Algorithms in Wireless Sensor Networks, International Journal of Computer Science \& Engineering Survey (IJCSES) 2 (4), (1205.1673). 